\title{
Lemierre Syndrome: Clinical Update and Protocol for a Systematic Review and Individual Patient Data Meta-analysis
}

\author{
Clara Sacco ${ }^{1}$ Federica Zane ${ }^{1} \quad$ Serena Granziera ${ }^{2,3}$ Karin Holm ${ }^{4}$ Dina Creemers-Schild ${ }^{5}$ \\ Michel-André Hotz ${ }^{6}$ Elena Turpini ${ }^{7} \quad$ Adele Valentini $^{7} \quad$ Christian Righini $^{8} \quad$ Petros D. Karkos ${ }^{9}$ \\ Peter Verhamme ${ }^{10}$ Marcello Di Nisio ${ }^{11}$ Stavros Konstantinides ${ }^{12,13}$ Alessandro Pecci ${ }^{1}$ \\ Stefano Barco ${ }^{12}$ on behalf of the Lemierre Study Group
}

${ }^{1}$ Department of Internal Medicine, IRCCS Policlinico San Matteo Foundation, University of Pavia, Pavia, Italy

2 Department of Medicine (DIMED), Geriatric Clinic, University of Padua, Padua, Italy

${ }^{3}$ Department of Physical and Rehabilitation Medicine, "Villa Salus" Hospital, Mestre, Italy

${ }^{4}$ Division of Infection Medicine, Department of Clinical Sciences, Lund University, Lund, Sweden

5 Department of Internal Medicine, Langeland Hospital, Zoetermeer, The Netherlands

${ }^{6}$ Department of ENT, Head and Neck Surgery, Inselspital (University Hospital of Bern), University of Bern, Bern, Switzerland

${ }^{7}$ Department of Radiology, IRCCS Policlinico San Matteo Foundation, University of Pavia, Pavia, Italy

${ }^{8}$ Department of Otolaryngology-Head and Neck Surgery, Grenoble University Hospital, Grenoble, France

${ }^{9}$ Department of Otolaryngology-Head and Neck Surgery, AHEPA Hospital, Aristotle University of Thessaloniki, Thessaloniki, Greece

10 Department of Vascular Medicine and Haemostasis, University Hospitals Leuven, Leuven, Belgium

${ }^{11}$ Department of Medicine and Ageing Sciences, University G. D'Annunzio of Chieti-Pescara, Chieti, Italy

${ }^{12}$ Center for Thrombosis and Haemostasis, University Medical Center of the Johannes Gutenberg University, Mainz, Germany

${ }^{13}$ Department of Cardiology, Democritus University of Thrace, Alexandroupolis, Greece

Hämostaseologie 2019;39:76-86.
Address for correspondence Stefano Barco, MD, PhD, Center for Thrombosis and Hemostasis, University Medical Hospital of the Johannes Gutenberg University, 403-131, Langenbeckstraße 1, 55131 Mainz, Germany (e-mail: s.barco@uni-mainz.de).

\author{
Abstract \\ Keywords \\ - Lemierre syndrome \\ - venous \\ thromboembolism \\ - bacterial infection \\ - anticoagulant \\ treatment \\ - Fusobacterium \\ necrophorum
}

Lemierre syndrome usually affects otherwise healthy adolescents or young adults and occurs at an overall rate of 1 to 10 cases per million person-years with an estimated fatality rate of 4 to $9 \%$. Diagnostic criteria remain debated and include acute neck/head bacterial infection (often tonsillitis caused by anaerobes at high potential for sepsis and vascular invasion, notably Fusobacterium necrophorum) complicated by local vein thrombosis, usually involving the internal jugular vein, and systemic septic embolism. Medical treatment is based on antibiotic therapy with anaerobic coverage, anticoagulant drugs and supportive care in case of sepsis. Surgical procedures can be required, including drainage of the abscesses, tissue debridement and jugular vein ligation. Evidence for clinical management is extremely poor in the absence of any adequately received

August 14, 2017

accepted after revision

March 9, 2018 (c) 2019 Georg Thieme Verlag KG Stuttgart · New York
DOI https://doi.org/ 10.1055/s-0038-1654720. ISSN 0720-9355. 
Zusammenfassung

\author{
Schlüsselwörter \\ - Lemierre-Syndrom \\ - Venöse \\ Thromboembolie \\ - bakterielle Infektion \\ - Antikoagulanz \\ - Fusobacterium \\ necrophorum
}

sized study with clinical outcomes. In this article, we illustrate two cases of Lemierre syndrome not caused by Fusobacterium necrophorum and provide a clinically oriented discussion on the main issues on epidemiology, pathophysiology and management strategies of this disorder. Finally, we summarize the study protocol of a proposed systematic review and individual patient data meta-analysis of the literature. Our ongoing work aims to investigate the risk of new thromboembolic events, major bleeding or death in patients diagnosed with Lemierre syndrome, and to better elucidate the role of anticoagulant therapy in this setting. This effort represents the starting point for an evidence-based treatment of Lemierre syndrome built on multinational interdisciplinary collaborative studies.

Das Lemierre-Syndrom betrifft normalerweise gesunde Jugendliche oder junge Erwachsene und tritt mit einer Gesamttodesrate von 1 bis 10 Fällen pro Million Personenjahre mit einer geschätzten Sterblichkeitsrate von 4 bis $9 \%$ auf. Diagnostische Kriterien bleiben umstritten und umfassen akute Kopf-Hals-Bakterieninfektionen (häufig Tonsillitis durch Anaerobier mit hohem Sepsis-und Gefäßinvasionspotenzial, insbesondere Fusobacterium necrophorum), kompliziert durch lokale Venenthrombose, die gewöhnlich die Vena jugularis interna involviert, und systemische septische Embolie. Die medizinische Behandlung basiert auf einer Antibiotikatherapie mit anaerober Abdeckung, Antikoagulanzien, und unterstützender Behandlung bei Sepsis. Chirurgische Maßnahmen können erforderlich sein, einschließlich der Drainage der Abszesse, Gewebedebridement und Jugularvenenligatur. Die Evidenz für das klinische Management ist in Ermangelung einer ausreichend bemessenen Studie mit klinischen Ergebnissen äußerst schlecht. In diesem Artikel illustrieren wir zwei Fälle von LemierreSyndrom, die nicht durch Fusobacterium necrophorum verursacht wurden und bieten eine klinisch orientierte Diskussion über die Hauptprobleme der Epidemiologie, Pathophysiologie und Managementstrategien dieser Erkrankung. Abschließend fassen wir das Studienprotokoll einer vorgeschlagenen systematischen Übersichtsarbeit und einer individuellen Patientendaten-Metaanalyse der Literatur zusammen. Unsere laufende Arbeit zielt darauf ab, das Risiko neuer thromboembolischer Ereignisse, schwerer Blutungen oder Todesfälle bei Patienten mit Lemierre-Syndrom zu untersuchen und die Rolle der Antikoagulanzien-Therapie in diesem Zusammenhang besser zu klären. Diese Bemühungen bilden den Ausgangspunkt für eine evidenzbasierte Behandlung des Lemierre-Syndroms, welche auf multinationalen interdisziplinären kollaborativen Studien aufbaut.

\section{Two Cases of Lemierre Syndrome: Does One Definition Fit All?}

\section{Case 1}

A previously healthy 23-year-old male was admitted for sore throat, neck pain, diarrhoea and vomiting starting 1 week earlier. Physical examination revealed left tonsil enlargement, neck swelling and chronic periodontitis. In the suspicion of bacterial sepsis (fever, tachycardia, high respiratory rate, leukocytosis $\left[16.1^{*} 10^{9} / \mathrm{L}\right]$, elevated inflammatory biomarkers [C-reactive protein $32.0 \mathrm{mg} / \mathrm{L}$; procalcitonin 276.7 $\mu \mathrm{g} / \mathrm{L}])$, the patient was started on piperacillin-tazobactam, levofloxacin, steroids and fluid resuscitation. Despite treatment, he developed severe hypotension, hypoxaemia, acute renal failure and thrombocytopenia. Neck compression ultrasound showed left internal jugular vein thrombosis; computed tomographic pulmonary angiography (CTPA) depicted left superior thyroid vein thrombosis and bilateral pulmonary abscesses (- Figs. 1 and 2). Antibiotic therapy was switched to meropenem plus metronidazole, and prophylactic-dose enoxaparin. A prophylactic dose of low molecular weight heparin (LMWH) was preferred in the presence of conditions reducing its clearance (renal failure) and increasing the individual risk of bleeding (thrombocytopenia). An anaerobic gram-negative rod of the Prevotella spp. was isolated from blood cultures. The patient's clinical condition progressively improved. Three weeks later, CTPA showed reduction in the size of the pulmonary nodules and resolution of jugular and thyroid vein thromboses. The patient was discharged on amoxicillin-clavulanate, 

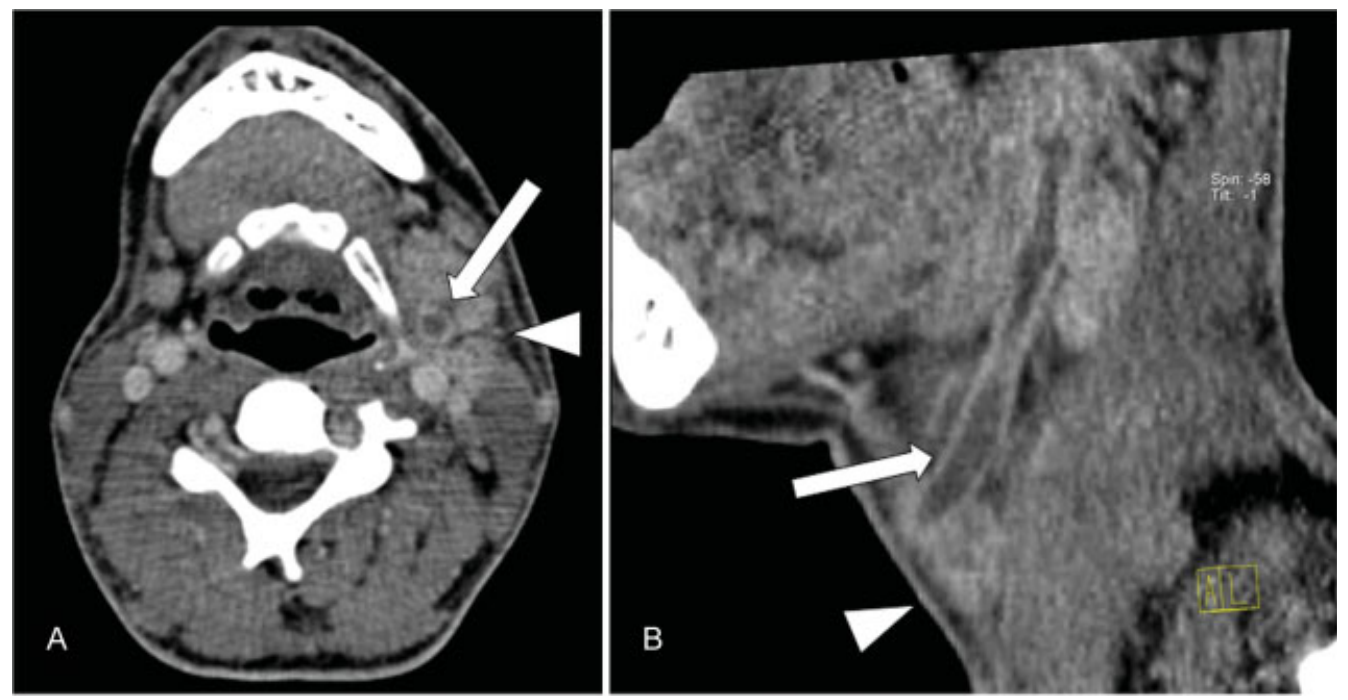

Fig. 1 Clinical case 1: Thrombosis of the left superior thyroid vein (arrow) associated with lymphadenomegaly of the left neck (arrowhead).

ciprofloxacin and prophylactic-dose enoxaparin, which was discontinued after 6 weeks from discharge after completion of approximately 3 months of treatment, without further complications.
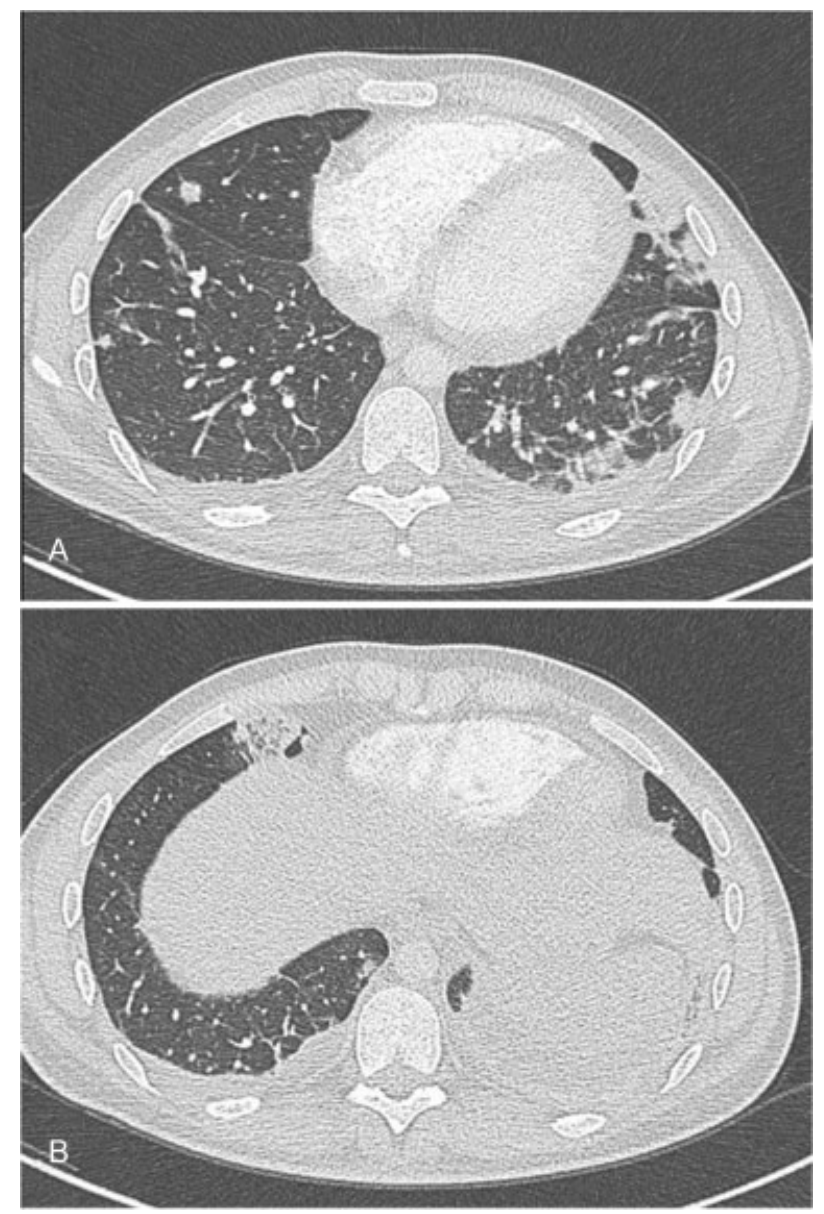

Fig. 2 Clinical case 1: Computer tomography pulmonary angiogram sections showing multiple bilateral nodules within the lung parenchyma consistent with infarctions evolving to abscesses (both panels).

\section{Case 2}

A 40-year-old male presented with a 5-day history of fever and neck swelling associated with leukocytosis $\left(20.1^{*} 10^{9} / \mathrm{L}\right)$ and elevated inflammatory biomarkers (C-reactive protein $20.4 \mathrm{mg} / \mathrm{L}$; procalcitonin $14.2 \mu \mathrm{g} / \mathrm{L}$ ). He reported chronic hepatitis B virus infection, hypertension, diabetes mellitus and recent tooth extraction. CTPA detected right internal jugular vein thrombosis, lymphadenomegaly, right laterocervical abscess and focal ground glass alterations in the ventral segment of the upper lobe of the right lung (-Fig. 3). Incision and drainage of the abscess was performed and the Gram-negative Klebsiella pneumoniae was isolated from cultures of the drainage material. The patient received meropenem, vancomycin and therapeutic-dose nadroparin. CTPA performed 6 weeks after admission demonstrated complete recovery of the previous alterations. The patient was discharged on trimethoprim-sulfamethoxazole and therapeutic-dose nadroparin. Anticoagulation was given for a total of 3 months without complications.

\section{Lemierre Syndrome: An Update}

\section{How to Define and Diagnose Lemierre Syndrome}

André Lemierre was among the first physicians to provide a comprehensive description of the clinical features of what he called 'anaerobic post-anginal septicaemia' in $1936,{ }^{1}$ named after him (but only 50 years later) 'Lemierre syndrome', ${ }^{2}$ or alternatively, with the synthetic genitive, 'Lemierre's syndrome'. He described the syndrome as 'usually affecting young adults or adolescents, with both sexes being equally attacked', sometimes occurring in small epidemics, with 'the most usual initial cause being a tonsillar or peritonsillar abscess, opened too late or to an insufficient degree'. Moreover, 'a simple tonsillitis may conceal small foci of suppuration. [...] These septicaemias are the result of a thrombophlebitis of the tonsillar and peritonsillar veins, which can spread to the internal jugular vein or even to the facial vein. [...]. The B. funduliformis septicaemias have always been accompanied 

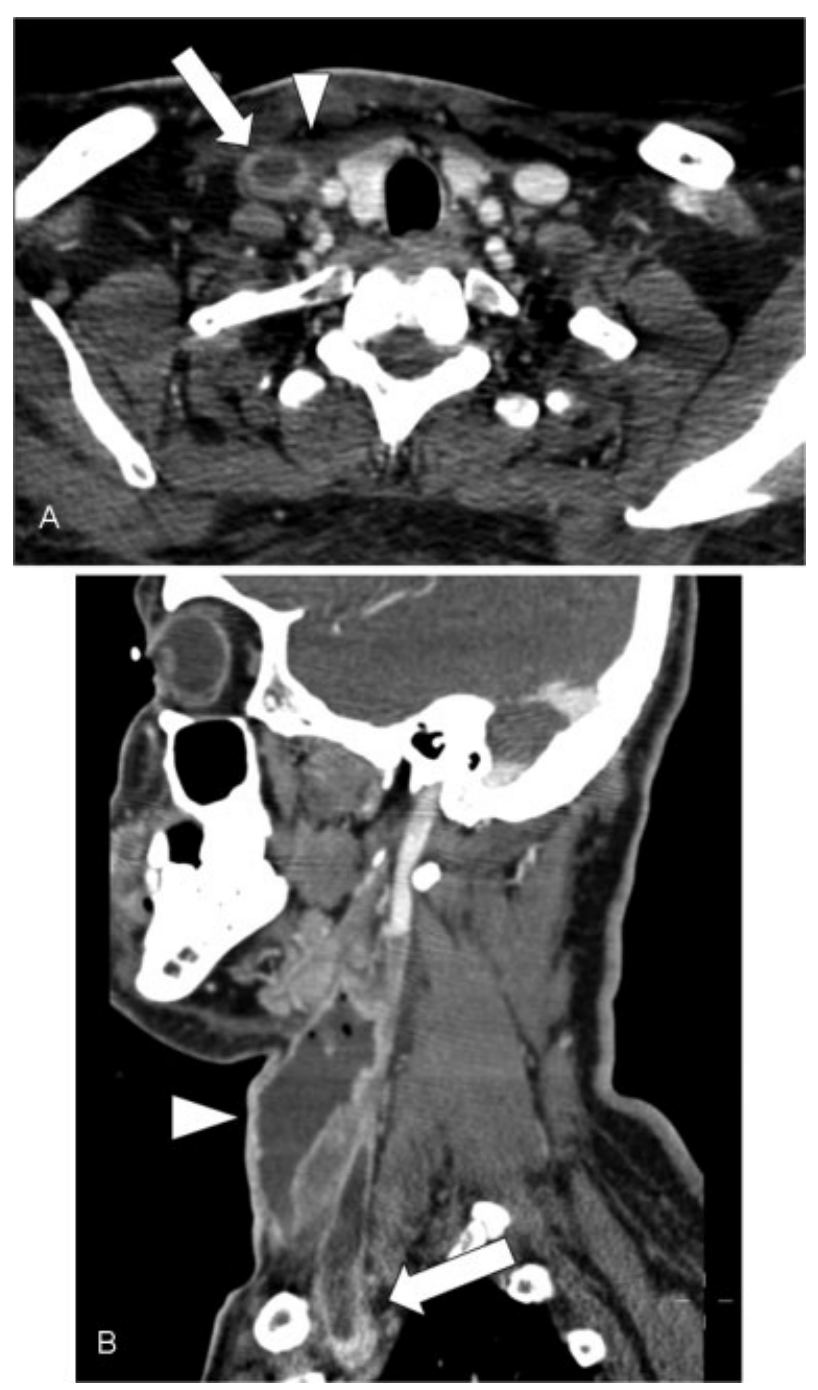

Fig. 3 Clinical case 2: Internal jugular vein thrombosis and large right laterocervical abscess. (A) Thrombosis of the right internal jugular vein (arrow). Contrast enhancement and thickening of the vessel wall indicates venous thrombosis associated with signs of inflammation and oedema of the perivascular adipose tissue (arrowhead).

(B) Thrombosis of the right internal jugular vein (arrow) and large right laterocervical abscess (arrowhead).

by the formation of distant metastatic abscesses', as well as by multiorgan failure, and can rapidly progress to death. ${ }^{1}$ Despite this early and clear recognition of a new nosological entity, there is still no agreement regarding the criteria required for confirming the diagnosis of Lemierre syndrome. ${ }^{3,4}$ This may partly explain the delayed introduction as a separate Medical Subject Heading (Unique MeSH) ID D057831 in 2011 and the persisting absence of a specific International Classification of Diseases code for its identification.

Several definitions, including the initial one made by Lemierre himself, pinpoint the varying characteristics of the vascular involvement (venous thrombosis and/or septic embolism), organ failure and anaerobic bacterial species detected from local source of infection or from an otherwise sterile site. Stricter criteria are suggested in the narrative review authored by Riordan in $2007,{ }^{5}$ which focused on a total of 222 cases, namely: (1) history of [tonsillar] anginal illness or compatible clinical findings; (2) evidence of metastatic lesions in lungs and/or another site; and (3) either evidence of internal jugular vein thrombophlebitis or isolation of $F$. necrophorum, a strict anaerobe Gram-negative rod, or Fusobacterium spp., from blood cultures or from a normally sterile site. As previously mentioned, more liberal definitions or variants are found in the literature, which include other head and neck infectious foci than tonsils and widely heterogeneous thromboembolic complications, ranging from cerebral sinus vein to carotid artery involvement, presenting alone or in association with internal jugular vein thrombosis. ${ }^{6}$ Furthermore, as highlighted by our cases and in previous reports, other anaerobic or aerobic bacteria, such as Prevotella spp. (Case 1$)^{7,8}$ or Klebsiella pneumonia (Case 2), ${ }^{9-12}$ are associated with similar clinical pictures. Whether these are truly causative agents, or $F$. necrophorum undetected, remains unknown. ${ }^{13,14}$ Importantly, differences in the definition of the diagnostic criteria of Lemierre syndrome do not ultimately translate to different diagnostic and therapeutic approaches.

The clinical suspicion of Lemierre syndrome should be raised-ideally before the signs of sepsis become manifestin teenagers or young adults presenting with sore throat, high temperature, chills and unilateral tenderness of the neck. This is especially the case if the rapid streptococcal antigen from a tonsillar swab is negative and in the presence of signs of multifocal pneumonia on a chest X-ray. A computed tomography (CT) scan of the neck and chest may facilitate the diagnosis of neck vein thrombosis and depict typical rounded multifocal infiltrates suggestive for septic pulmonary emboli.

An algorithm consisting of the sequential application of a clinical decision score, D-dimer testing and ultrasonography has been validated for the diagnosis of upper-extremity deep venous thrombosis, including jugular vein thrombosis; however, patients with abscesses and massive lymphadenomegaly, such as those with Lemierre syndrome, were underrepresented in the derivation study. ${ }^{15}$ The use of positron emission tomography with 2-[fluorine-18]fluoro-D-glucose integrated with CT (FDG-PET/CT) has been described in three patients with Lemierre syndrome and may represent an alternative or adjunct to conventional imaging in selected patients. ${ }^{16}$ Blood cultures usually confirm the diagnosis, being positive for anaerobic bacteria in most cases, together with a tonsillar swab cultured in selective anaerobic plates. Specific molecular detection using polymerase chain reaction techniques can add useful information. ${ }^{17}$

\section{Incidence and Case Fatality Rates}

Only a few studies have investigated the epidemiology of Lemierre syndrome, which remains an elusive disease because of its rarity. All the same, Lemierre syndrome is not 'a forgotten disease', as previously thought and often referred to. ${ }^{18}$ Clinical experience suggests that the incidence of Lemierre syndrome may be on the rise, and it remains to be seen whether this is due to antibiotic resistance, changes in antibiotic prescription patterns, more efficient diagnostics and/or publication trends ( - Fig. 4).

The most important prospective epidemiological study was conducted in Denmark between 1998 and 2001; it 


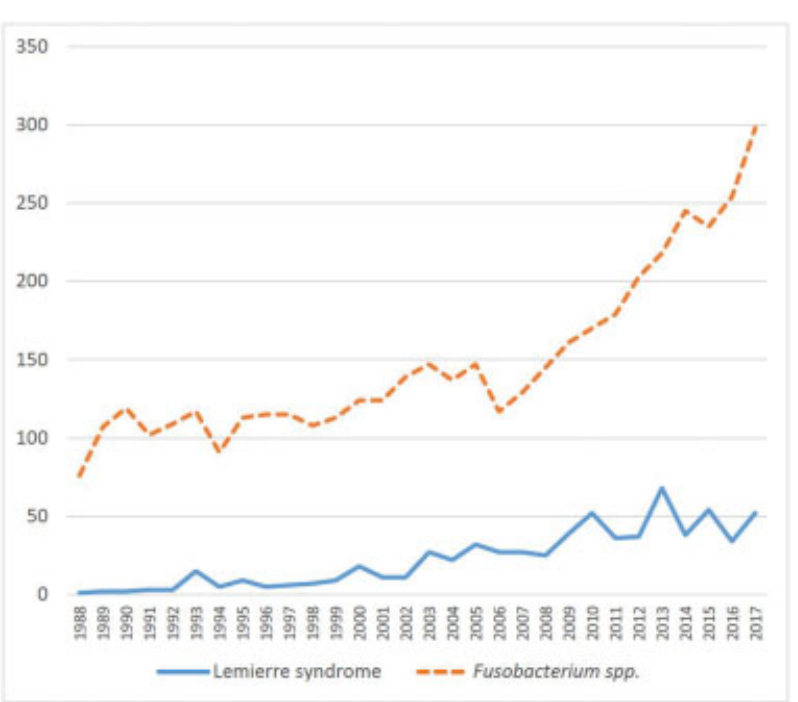

Fig. 4 Publication trends illustrating the annual number of studies reported in PubMed for Lemierre syndrome and Fusobacterium spp. between years 1988 and 2017.

estimated an annualized incidence rate of 3.6 cases per million in the general population. ${ }^{19}$ In this nationwide study, the authors included all disseminated $F$. necrophorum infection cases with primary foci in the head and neck: the highest incidence was found in individuals aged 15 to 24 years old with 14.4 cases per million/year, while the disorder was rare in adults $\geq 40$ years old. Previous reports suggested lower annualized incidence of approximately one event per million general population. ${ }^{20,21}$

A second Danish prospective analysis was conducted between 2010 and 2014, and focused on the incidence of F. necrophorum bacteraemia from microbiology samples. ${ }^{22}$ Consistently with what was previously reported, the estimated annualized incidence was higher in young individuals (9.4 cases per million patient-years) compared with children and middle-aged patients. With an overall estimated annualised incidence of 13.9 cases per million patient-years, they suggested an unchanged incidence in Denmark over the past two decades. ${ }^{22}$ Finally, some narrative reviews suggested higher probability of the syndrome in males with a $2: 1$ ratio, ${ }^{5,23,24}$ which was, however, not confirmed by others, ${ }^{25}$ with seasonal peaks in early autumn and late winter. $^{3}$

The overall case fatality rate was $9 \%$ with peaks of $26 \%$ in the elderly. ${ }^{19}$ The elevated risk of death in the elderly was subsequently confirmed.22 A narrative review of the literature attempting to focus on deaths unrelated to underlying disease, such as immunodeficiency or cancers, estimated an overall case fatality rate of 4 to $12 \%,{ }^{26}$ in line with what was observed (5\%) in a systematic review of 84 cases. $^{3}$ Indeed, one should be cautious in interpreting results based on a post hoc categorization of the causes of death.

\section{Causes of Acute Vein Thrombosis in Lemierre Syndrome}

Adult patients with acute bacterial infections have an approximately threefold increased risk of developing venous thromboembolism compared with patients without infection, especially within the first 2 weeks after onset. ${ }^{27,28}$ However, the absolute rate of thromboembolic complications remains low overall and no other infectious condition apart from the Lemierre syndrome presents with thromboembolic complications as an integral part of the initial clinical picture and diagnostic criteria. The jugular vein is commonly affected, likely due to its location in the parapharyngeal space adjacent to the tonsils. ${ }^{3,5}$ The thrombosis may be found also in the distal part of the jugular vein, and sometimes in the external jugular vein. ${ }^{6}$ It is therefore likely that the infection spreads not only via the tonsillar vein (into the lingual and pharyngeal veins, and into the internal jugular vein), but also through the tissues or via the lymphatic system leading to inflammation of the vessel wall, acute thrombosis and bacterial invasion.

F. necrophorum, the most common pathogen in Lemierre syndrome, has been shown to assemble and activate the procoagulant and pro-inflammatory contact system on its surface, leading to activation of the intrinsic pathway of coagulation and release of bradykinin, which in turn may favour thrombus stabilization and cause increased vascular leakage. ${ }^{29}$ F. necrophorum also binds to plasminogen, which is more readily activated to plasmin at the bacterial surface than unbound plasminogen; moreover, the plasmin bound to the surface is protected from inactivation by $\alpha 2$-antiplasmin. ${ }^{30}$ This can lead to increased invasion through extracellular tissue and adherence to endothelial cells. ${ }^{31}$ Indeed, many other pathogenic bacteria that are able to directly promote thrombosis, such as Staphylococcus aureus, ${ }^{32}$ can activate plasminogen, breach tissue barrier and more effectively spread and evade innate immune defense, ${ }^{33}$ supporting the concept of Lemierre syndrome not being exclusively caused by $F$. necrophorum.

In addition to Lemierre syndrome, the presence of F. necrophorum is also associated with localized pharyngotonsillitis and peritonsillar abscesses, preferably occurring in the same young age group. ${ }^{34,35}$ The causes of age distribution of Lemierre syndrome could involve a recent acquisition of the bacterium combined with individual lack of adaptive immunity, much in the same way as meningococcal disease affects non-immune teenagers worldwide. ${ }^{36}$ In addition to the adaptive immunity, modulation of innate immunity by the bacteria may be important for the invasion to occur. Most $F$. necrophorum strains are able to bind factor $\mathrm{H}$ to their surfaces and in a plasma environment this may contribute to the protection against complement attack by preventing (or limiting) complement activation. ${ }^{37}$ Moreover, F. necrophorum isolated from animals, and also found in human isolates, ${ }^{38,39}$ has long been known to secrete a leukotoxin that kills neutrophils and is considered a major virulence factor in animal disease due to $F$. necrophorum. ${ }^{40}$ Theoretically, it may impair local innate immunity and increase the invasiveness of the bacterium.

A recent Swedish study found that in two-thirds of the F. necrophorum positive pharyngotonsillitis patients, another bacterium or virus was also detected. ${ }^{41}$ In a cohort of 22 patients with Lemierre syndrome, the prevalence of serologically confirmed active Epstein-Barr virus infection 
was not higher (4.5\%) than one would expect in a paediatric population with pharingotonsillitis. ${ }^{6}$

In addition to pro-coagulant properties of the bacteria, host factors might also play a role in the genesis of thrombosis. The screening of 26 patients with Lemierre syndrome provided no conclusive data regarding the possible role of underlying thrombophilia. ${ }^{6}$

\section{How the Clinical Presentation of Lemierre Syndrome Influences Prognosis}

As discussed, the most frequent characteristics of Lemierre syndrome are a history of recent oropharyngeal infection, clinical or radiological evidence of neck vein thrombosis and isolation of usually anaerobic pathogens. However, there is often a fourth feature, the presence of distant metastatic emboli, associated or not with severe sepsis and septic shock, that raises the alarm and points towards the diagnosis. ${ }^{3}$ Neck vein thrombosis can lead to septic embolization via haematogenous spread. Lung involvement, followed by septic arthritis/osteomyelitis, deep neck space infections and liver, spleen, kidney and brain embolization are the most common manifestations affecting distant organs. ${ }^{3,5}$ If not diagnosed and 'interrupted' early, this phenomenon of distant bacterial spread increases morbidity and mortality and directly affects prognosis propagating the thrombo-inflammatory process leading to multiorgan failure.

Blood cultures can often be negative or take as long as 7 days for identification of $F$. necrophorum in less advanced bacteriological laboratories, ${ }^{42}$ and the same goes for tonsillar cultures. Imaging tests showing neck vein thrombosis or signs of septic embolism often provide the initial and only clue for suspecting Lemierre syndrome. A history of oropharyngeal infection, especially in previously healthy young adults or children, not responding to 'conventional' antibiotics within 24 hours of intravenous treatment, should alert the clinician for a multidisciplinary team approach. Early surgical treatment, when indicated, and aggressive intravenous antibiotics targeting anaerobes appear reasonable therapeutic options if any of the diagnostic features of Lemierre syndrome are present. As for severe sepsis and septic shock, ${ }^{43}$ the prognosis of Lemierre syndrome may largely depend on timely initial management, which should be ideally completed within 6 hours after the patient's presentation to minimize adverse outcomes. ${ }^{44,45}$ In a recent study of more than 49,000 patients with sepsis and septic shock, a rapid completion of a 3-hour course of sepsis care and administration of antibiotics, but not rapid completion of an initial bolus of intravenous fluids, were associated with lower mortality. ${ }^{46}$

\section{Controversy over the Benefits versus Risks of Anticoagulants}

The efficacy and safety of anticoagulant drugs for the treatment of vein thrombosis associated with Lemierre syndrome are uncertain. ${ }^{47}$ While prompt institution of anticoagulation may prevent thrombotic extension and embolization and new thromboembolic events, this treatment may expose patients to high risk of bleeding complications in the presence of concomitant bleeding risk factors (e.g. thrombocytopenia or worsening renal function due to sepsis). Moreover, it remains unclear whether anticoagulation may facilitate the spread of septic material and/or haemorrhagic transformation of embolic lesions, as it may be the case for the initiation of de novo anticoagulation in patients with infectious endocarditis. ${ }^{48}$ The limited evidence on the pharmacokinetics of anticoagulants in childhood or adolescence represents an additional challenge, although new therapeutic options and regimens are being studied, especially for long-term or extended treatment. ${ }^{49-52}$

When deciding on the agent and optimal dosing, physicians should carefully consider drug pharmacokinetics, individual patient characteristic, disease severity, bleeding risk and individual risk of new thromboembolic complications. For instance, it appears reasonable to prefer LMWH over fondaparinux in patients with moderate renal impairment because of their shorter half-life and the lower renal clearance. In the acute phase, unfractionated heparin may be considered in case of severe renal insufficiency and in the management of sepsis, since progression to severe sepsis and septic shock may be swift. The presence of mucosal lesions or inflammation, nausea and vomiting may decrease oral anticoagulant intake while concomitant or ensuing gastrointestinal disturbances (e.g. diarrhoea) may affect drug absorption.

Patients with clinical improvement may be transitioned to oral anticoagulation, which can be continued until up to 3 months as recommended by major clinical guidelines for the cases of provoked venous thrombosis. ${ }^{53}$ Longer courses of oral anticoagulation with either vitamin $\mathrm{K}$ antagonists or novel direct oral anticoagulants should be considered for patients who respond poorly (i.e. in terms of thrombus resolution), remain symptomatic despite antibiotic treatment or in whom the infectious process is not completely resolved. A periodic re-assessment of the risks of bleeding and recurrent thrombosis is warranted to decide on the optimal treatment duration, ${ }^{53}$ although no validated risk assessment models are available for the paediatric population and several of the predictors identified in adults, such as arterial hypertension, cancer and chronic renal dysfunction, would not apply.

\section{Designing and Conducting a Systematic Review and Individual Patient Data Meta-Analysis}

\section{Review Questions}

1. What is the rate of thromboembolic recurrence, thrombotic extension of index thrombosis or new embolism in patients diagnosed with Lemierre syndrome?

2. What are the major bleeding and fatality rates in patients diagnosed with Lemierre syndrome?

3. What are the estimated efficacy and safety of anticoagulant treatment?

\section{Eligibility Criteria}

The following diagnostic criteria for Lemierre syndrome served for the selection of potentially eligible studies: 
Table 1 Summary of the study protocol of the systematic review and individual patient data meta-analysis

\begin{tabular}{|c|c|}
\hline \multirow[t]{3}{*}{ Research questions } & $\begin{array}{l}\text { 1. What is the rate of thromboembolic recurrence, thrombotic extension of index thrombosis, or } \\
\text { new embolism in patients diagnosed with Lemierre syndrome? }\end{array}$ \\
\hline & 2. What is the rate of major bleeding and fatality rate in patients diagnosed with Lemierre syndrome? \\
\hline & 3. What are the estimated efficacy and safety of anticoagulant treatment? \\
\hline \multirow{3}{*}{$\begin{array}{l}\text { Diagnostic criteria } \\
\text { accepted for Lemierre } \\
\text { syndrome }\end{array}$} & - The primary site of infection being located at either the head or the neck sites \\
\hline & $\begin{array}{l}\text { - Isolation of } F \text {. necrophorum or other bacterial organism potentially causing Lemierre syndrome, } \\
\text { associated or not with the presence of sepsis }\end{array}$ \\
\hline & - Neck/cerebral/upper-extremity thrombosis and/or septic embolism \\
\hline \multirow[t]{3}{*}{ Study outcomes ${ }^{\mathrm{a}}$} & $\begin{array}{l}\text { - Recurrent venous thromboembolism (new thrombotic events or extension/recurrence), and new } \\
\text { septic embolism }\end{array}$ \\
\hline & - Major and non-major bleeding (ISTH criteria) ${ }^{55}$ \\
\hline & - Death from all causes \\
\hline \multirow[t]{6}{*}{ Subgroup analyses } & - Paediatric versus adult population \\
\hline & - Patients grouped by use and type of anticoagulant agent \\
\hline & - Patients undergoing surgery versus not \\
\hline & - Type of antibiotic treatment received and type of bacteria involved \\
\hline & - By geographical region \\
\hline & - Based on the results of quality assessment \\
\hline $\begin{array}{l}\text { Registration of the } \\
\text { study protocol }\end{array}$ & International prospective register of systematic reviews PROSPERO (ID CRD42016052572) \\
\hline
\end{tabular}

Abbreviation: ISTH, International Society on Thrombosis and Haemostasis.

${ }^{\mathrm{a}}$ Assessment of all outcomes is considered either during hospitalization or at a short-term follow-up (30 days).

(1) the primary site of infection being located at either the head or the neck sites; (2) isolation of $F$. necrophorum or other bacterial organism potentially causing Lemierre syndrome, associated or not with the presence of sepsis; and (3) neck/cerebral/upper-extremity thrombosis and/or septic embolism ( - Table 1). This definition of Lemierre syndrome aims to focus on a broad spectrum of cases originated from head/neck bacterial infections and associated with characteristics thromboembolic complications to perform predefined analyses in relevant patient sub-groups. Such definition does not cover cases originated from sub-diaphragmatic infections (i.e. urogenital or gastrointestinal infections, or liver abscesses) since septic acute thrombosis usually affects older individuals and might be due to different etiopathology, including impaired mucosal integrity due to cancer. ${ }^{54}$

Literature search covered the period elapsing from January 2000 (week 1) to October 2016 (week 3). Only studies published after year 1999 were included since that date approximately represents the time since LMWH became commonly prescribed worldwide for parenteral anticoagulation. We applied neither language nor study design restrictions to the literature search, as we anticipated that the vast majority of studies consist of case reports or small case series.

\section{Literature Search Strategy and Data Extraction}

We systematically searched MEDLINE (via PubMed), Embase (via Ovid) and the Cochrane Library database on 19 October 2016 ( - Table 2). Our literature search identified a total of
1,971 studies ( $n=1,577$ for the timeframe selected and $n=1,012$ after removing duplicates). Details of our literature search strategy are provided in the - Supplementary Material. We complemented this search by manually reviewing references of retrieved articles, review papers, guidelines and grey literature (Google Scholar, ResearchGate). In case of duplicates or multiple congress abstracts, we decided to use the most recent full-text paper.

Titles and abstracts were screened by three reviewers, who selected titles subsequently assessed for eligibility after evaluation of full-texts. Disagreements were solved by consensus. Neither of the reviewers was blind to the journal titles or to the study authors or institutions. Corresponding authors were contacted by email within at least 2 weeks apart to provide missing data or to clear ambiguity about original data, if crucial information were lacking and to retrieve additional information regarding the outcomes of interest.

The following variables were collected: age, sex, year, type of article and number of patients included, cancer (presence and type), infection of head and/or neck (presence and type), isolation of $F$. necrophorum, isolation of other pathogens (type or phyla), utilization and type of antibiotics, evidence of jugular vein thrombosis at the time of Lemierre syndrome diagnosis, thrombosis in other head/neck districts, description and treatment of first event of thrombosis (yes/no, kind of anticoagulant treatment, dosage, days of treatment), clinical outcomes (occurrence, type of anticoagulant treatment, time to event), surgical treatment, delay in diagnosis and length of hospitalization. 


\section{Study Outcomes}

Recurrent thromboembolic events were defined as new thrombotic events or extension/recurrent thrombosis occurring at the same site (-Table 1 ). The occurrence of new septic embolism was defined on the basis of the description provided in the original paper. Bleeding events were retrospectively classified as 'major' or 'non-major' (International Society on Thrombosis and Haemostasis criteria). ${ }^{55}$ Major bleeding includes fatal bleeding, and/or symptomatic bleeding in a critical area or organ, such as intra-cranial, intraspinal, intra-ocular, retroperitoneal, intra-articular or pericardial or intra-muscular with compartment syndrome, and/ or bleeding causing a fall in haemoglobin level of $20 \mathrm{~g} \mathrm{~L}^{-1}$ or more, or leading to transfusion of two or more units of whole blood or red cells. Reasons of death are categorized as Lemierre syndrome-related (sepsis, thromboembolic/bleeding complications, post-surgical complications) or not. Assessment of all outcomes was considered either during hospitalization or at a short-term follow-up (30 days). Information on all outcomes was collected in a separate case report form prepared by a third investigator and adjudicated independently based on the aforementioned criteria by two investigators unaware of the anticoagulant treatment status described in the original studies.

\section{Quality Assessment and Risk of Bias}

The included studies were primarily categorized according with their study design (case reports, small series of cases, cohort studies), the presence of consecutive patients and the proportion of missing data. To minimize the risk of publication bias, all the authors of the included studies are contacted and asked whether some unspecified selection criteria were applied to the published report and if they have treated other patients with Lemierre syndrome before, after or concomitantly to the current publication (-Table 2). As previously mentioned, relevant papers in the field and grey literature were screened to detect additional publications, since traditional databases may not include all case reports, particularly those not undergoing a peer-review process. ${ }^{56}$

\section{Registration of the Study Protocol and Status}

The study is registered in the international prospective register of systematic reviews PROSPERO (available at crd. york.ac.uk/PROSPERO) on 1 December 2016 and updated on 10 August 2017 for status of the review and minor corrections (ID CRD42016052572; - Table 2). After completion of data retrieval and quality assessment, we expect to complete the primary analysis by June 2018 .

\section{Statistical Analysis}

Aggregate data will be analysed at the level of individual patients and a quantitative synthesis is planned. Rates of events occurring within 30 days (or in-hospital) will be provided as proportions and 95\% confidence intervals. Predictors of 30-day adverse outcomes will be evaluated in exploratory analyses by logistic regression models. Sensitivity analyses accounting for studies grouped according with study design, quality assessment and proportion of missing data are planned. Predefined sub-group analyses will include: (1) paediatric versus adult population; (2) patients grouped by type of anticoagulant agent; (3) patients undergoing surgery versus not; (4) type of antibiotic treatment received and type of bacteria involved; (5) by geographical region; and (6) based on the results of quality assessment ( - Table 1). Multiple imputation techniques will be considered for overcoming the problem of cases with missing values.

The general methodology for performing case reports (or cases series)-based analyses has been adapted from available studies with similar design and aims investigating rare

Table 2 Phases of the systematic review and patient level analysis

\begin{tabular}{|l|l|}
\hline \multirow{5}{*}{ Study design } & $\begin{array}{l}\text { Definition of the research questions, study population of interest and study outcomes (study } \\
\text { protocol and registration) }\end{array}$ \\
\cline { 2 - 3 } & Development of the electronic database for data extraction \\
\cline { 2 - 3 } & Development of the literature search strategy \\
\cline { 2 - 3 } & Literature search in PubMed, Embase and the Cochrane Collaboration repository \\
\hline \multirow{5}{*}{ Selection of studies } & Evaluation for eligibility based on titles and abstracts \\
\cline { 2 - 3 } & Retrieval of full texts of the selected studies \\
\cline { 2 - 3 } & Second selection based on full texts \\
\cline { 2 - 3 } & Retrieval of additional studies from grey literature and cross-referencing \\
\cline { 2 - 3 } & Data extraction \\
\hline Data ansessment & Quality assessment of the included studies \\
\cline { 2 - 3 } & $\begin{array}{l}\text { E-mail contact with all the authors of the studies to provide an estimate of publication bias and to } \\
\text { retrieve missing data }\end{array}$ \\
\hline & Data cleaning \\
\cline { 2 - 2 } & Adjudication of the study outcomes \\
\cline { 2 - 2 } & Statistical analysis \\
\hline
\end{tabular}


conditions. ${ }^{57-62}$ The recommendations listed in the Preferred Reporting Items for Systematic Review and Meta-Analyses of individual patient data statement will be followed, where applicable, for reporting the results of this analysis. ${ }^{63}$

\section{Conclusions and Future Outlook}

Although its identification and general definition date back to the beginning of the past century, Lemierre syndrome has never been the object of structured large-scale clinical research. Therefore, no specific evidence supports the clinical management of these patients, which is otherwise pawned by recent diagnostic and therapeutic advances for the treatment of other infectious and thrombotic conditions. Main reasons for this are the lack of definite diagnostic criteria, poor general awareness and intrinsic difficulties in setting up an adequate structure for implementing multicentre collaborative studies for rare diseases, as well as in-hospital management being often spread among various medical specialties according to the individual clinical presentation.

The proposed systematic review and individual patient data meta-analysis of the literature aim to document and summarize the current available knowledge derived from studies published in the past two decades, and provide a first quantitative analysis of clinically relevant outcomes, also related to anticoagulant treatment. This effort represents the obligatory starting point for setting up large multinational interdisciplinary studies in the field.

\section{Authors' Contributions}

Clara Sacco, Federica Zane, Serena Granziera, Marcello Di Nisio and Alessandro Pecci were responsible for the design of the study, collection of data, writing of the manuscript and gave final approval. Karin Holm and Petros Karkos were responsible for the writing of the manuscript, critical revision of the manuscript and gave final approval. Elena Turpini and Adele Valentini were responsible for collection of data, critical revision of the manuscript and gave final approval. Dina Creemers-Schild, Michael-André Hotz, Christian Righini, Peter Verhamme and Stavros Konstantinides critically revised the manuscript and gave final approval. Stefano Barco was responsible for the concept and design of the study, collection of data, quality assessment and data analysis, writing of the manuscript and gave final approval.

\section{Conflicts of Interest}

Marcello Di Nisio reports personal fees from Daiichi and Bayer HealthCare. Stavros Konstantinides has received consultancy, advisory board and lecture fees from Bayer HealthCare, Boehringer Ingelheim, Merck Sharp \& Dohme, Daiichi-Sankyo, Pfizer-Bristol-Myers Squibb and BTG Biocompatibles Group UK; and institutional grants from Boehringer Ingelheim, Bayer HealthCare, Daiichi-Sankyo, Merck Sharp \& Dohme-Pfizer and Actelion. All the other authors do not report any potential conflict of interest for the present publication.
The work of Stavros Konstantinides and Stefano Barco is supported by the German Federal Ministry of Education and Research (BMBF 01E01003 and 01E01503).

\section{References}

1 Lemierre A. On certain septicaemias due to anaerobic organisms. Lancet 1936;1:701-703

2 Shannon GW, Ellis CV, Stepp WP. Oropharyngeal bacteroides melaninogenicus infection with septicemia: Lemierre's syndrome. J Fam Pract 1983;16(01):159-160, 163, 166

3 Karkos PD, Asrani S, Karkos CD, et al. Lemierre's syndrome: a systematic review. Laryngoscope 2009;119(08):1552-1559

4 Syed MI, Baring D, Murray C. In reference to Lemierre's syndrome: a systematic review. Laryngoscope 2010;120(01):215-216, author reply 216

5 Riordan T. Human infection with Fusobacterium necrophorum (Necrobacillosis), with a focus on Lemierre's syndrome. Clin Microbiol Rev 2007;20(04):622-659

6 Holm K, Svensson PJ, Rasmussen M. Invasive Fusobacterium necrophorum infections and Lemièrre's syndrome: the role of thrombophilia and EBV. Eur J Clin Microbiol Infect Dis 2015 34(11):2199-2207

7 Wani P, Antony N, Wardi M, Rodriguez-Castro CE, Teleb M. The forgotten one: Lemierre's syndrome due to Gram-negative rods Prevotella bacteremia. Am J Case Rep 2016;17:18-22

8 Huits RM, van Assen S, Wildeboer-Veloo AC, Verschuuren EA, Koeter GH. Prevotella bivia necrobacillosis following infectious mononucleosis. J Infect 2006;53(02):e59-e63

9 Chuncharunee A, Khawcharoenporn T. Lemierre's syndrome caused by Klebsiella pneumoniae in a diabetic patient: a case report and review of the literature. Hawaii J Med Public Health 2015;74(08):260-266

10 Garbati MA, Ahsan AM, Hakawi AM. Lemierre's syndrome due to Klebsiella pneumoniae in a 63-year-old man with diabetes: a case report. J Med Case Reports 2012;6:97

11 Lee WS, Wang FD, Shieh YH, Teng SO, Ou TY. Lemierre syndrome complicating multiple brain abscesses caused by extended-spectrum $\beta$-lactamase-producing Klebsiella pneumoniae cured by fosfomycin and meropenem combination therapy. J Microbiol Immunol Infect 2012;45(01):72-74

12 Tsai YJ, Lin YC, Harnnd DJ, Chiang RP, Wu HM. A Lemierre syndrome variant caused by Klebsiella pneumoniae. J Formos Med Assoc 2012;111(07):403-405

13 Osman M, Hasan S, Bachuwa G. Oesophageal cancer presenting as Lemierre's syndrome caused by Streptococcus anginosus. BMJ Case Rep 2017;2017:bcr-2017-bcr-219661

14 Alberio L, Lämmle B. Images in clinical medicine. Capnocytophaga canimorsus sepsis. N Engl J Med 1998;339(25):1827

15 Kleinjan A, Di Nisio M, Beyer-Westendorf J, et al. Safety and feasibility of a diagnostic algorithm combining clinical probability, D-dimer testing, and ultrasonography for suspected upper extremity deep venous thrombosis: a prospective management study. Ann Intern Med 2014;160(07):451-457

16 Pijl JP, Glaudemans AWJM, Slart RHJA, Kwee TC. FDG-PET/CT as a new method for diagnosis and whole-body evaluation of Lemierre syndrome. Clin Nucl Med 2017;42(08):e377-e380

17 Aliyu SH, Yong PF, Newport MJ, et al. Molecular diagnosis of Fusobacterium necrophorum infection (Lemierre's syndrome). Eur J Clin Microbiol Infect Dis 2005;24(03):226-229

18 Weesner CL, Cisek JE. Lemierre syndrome: the forgotten disease. Ann Emerg Med 1993;22(02):256-258

19 Hagelskjaer Kristensen L, Prag J. Lemierre's syndrome and other disseminated Fusobacterium necrophorum infections in Denmark: a prospective epidemiological and clinical survey. Eur J Clin Microbiol Infect Dis 2008;27(09):779-789 
20 Hagelskjaer LH, Prag J, Malczynski J, Kristensen JH. Incidence and clinical epidemiology of necrobacillosis, including Lemierre's syndrome, in Denmark 1990-1995. Eur J Clin Microbiol Infect Dis 1998;17(08):561-565

21 Jones JW, Riordan T, Morgan MS. Investigation of postanginal sepsis and Lemierre's syndrome in the South West Peninsula. Commun Dis Public Health 2001;4(04):278-281

22 Bank S, Jensen A, Nielsen HM, Kristensen LH, Voldstedlund M, Prag J. Fusobacterium necrophorum findings in Denmark from 2010 to 2014 using data from the Danish microbiology database. APMIS 2016;124(12):1087-1092

23 Brazier JS, Hall V, Yusuf E, Duerden BI. Fusobacterium necrophorum infections in England and Wales 1990-2000. J Med Microbiol 2002;51(03):269-272

24 Leugers CM, Clover R. Lemierre syndrome: postanginal sepsis. J Am Board Fam Pract 1995;8(05):384-391

25 Armstrong AW, Spooner K, Sanders JW. Lemierre's syndrome. Curr Infect Dis Rep 2000;2(02):168-173

26 Chirinos JA, Lichtstein DM, Garcia J, Tamariz LJ. The evolution of Lemierre syndrome: report of 2 cases and review of the literature. Medicine (Baltimore) 2002;81(06):458-465

27 Schmidt M, Horvath-Puho E, Thomsen RW, Smeeth L, Sørensen HT. Acute infections and venous thromboembolism. J Intern Med 2012;271(06):608-618

28 Smeeth L, Cook C, Thomas S, Hall AJ, Hubbard R, Vallance P. Risk of deep vein thrombosis and pulmonary embolism after acute infection in a community setting. Lancet 2006;367(9516): 1075-1079

29 Holm K, Frick IM, Björck L, Rasmussen M. Activation of the contact system at the surface of Fusobacterium necrophorum represents a possible virulence mechanism in Lemièrre's syndrome. Infect Immun 2011;79(08):3284-3290

30 Holm K, Rasmussen M. Binding and activation of plasminogen at the surface of Fusobacterium necrophorum. Microb Pathog 201359-60:29-32

31 Kumar A, Menon S, Nagaraja TG, Narayanan S. Identification of an outer membrane protein of Fusobacterium necrophorum subsp. necrophorum that binds with high affinity to bovine endothelial cells. Vet Microbiol 2015;176(1-2):196-201

32 Liesenborghs L, Verhamme P, Vanassche T. Staphylococcus aureus, master manipulator of the human hemostatic system. J Thromb Haemost 2018;16(03):441-454

33 Peetermans M, Vanassche T, Liesenborghs L, Lijnen RH, Verhamme P. Bacterial pathogens activate plasminogen to breach tissue barriers and escape from innate immunity. Crit Rev Microbiol 2016;42(06):866-882

34 Holm K, Bank S, Nielsen H, Kristensen LH, Prag J, Jensen A. The role of Fusobacterium necrophorum in pharyngotonsillitis - a review. Anaerobe 2016;42:89-97

35 Rusan M, Klug TE, Ovesen T. An overview of the microbiology of acute ear, nose and throat infections requiring hospitalisation. Eur J Clin Microbiol Infect Dis 2009;28(03):243-251

36 Trotter C, Borrow R, Andrews N, Miller E. Seroprevalence of meningococcal serogroup $C$ bactericidal antibody in England and Wales in the pre-vaccination era. Vaccine 2003;21(11-12): 1094-1098

37 Friberg N, Carlson P, Kentala E, et al. Factor $\mathrm{H}$ binding as a complement evasion mechanism for an anaerobic pathogen, Fusobacterium necrophorum. J Immunol 2008;181(12): 8624-8632

38 Holm K, Collin M, Hagelskjær-Kristensen L, Jensen A, Rasmussen $M$. Three variants of the leukotoxin gene in human isolates of Fusobacterium necrophorum subspecies funduliforme. Anaerobe 2017;45:129-132

39 Tadepalli S, Stewart GC, Nagaraja TG, Narayanan SK. Human Fusobacterium necrophorum strains have a leukotoxin gene and exhibit leukotoxic activity. J Med Microbiol 2008;57(Pt 2): $225-231$
40 Narayanan SK, Nagaraja TG, Chengappa MM, Stewart GC. Leukotoxins of gram-negative bacteria. Vet Microbiol 2002;84(04): 337-356

41 Hedin K, Bieber L, Lindh M, Sundqvist M. The aetiology of pharyngotonsillitis in adolescents and adults - Fusobacterium necrophorum is commonly found. Clin Microbiol Infect 2015 21(03):263.e1-263.e7

42 Bondy P, Grant T. Lemierre's syndrome: what are the roles for anticoagulation and long-term antibiotic therapy? Ann Otol Rhinol Laryngol 2008;117(09):679-683

43 Angus DC, van der Poll T. Severe sepsis and septic shock. N Engl J Med 2013;369(09):840-851

44 Levy MM, Dellinger RP, Townsend SR, et al; Surviving Sepsis Campaign. The Surviving Sepsis Campaign: results of an international guideline-based performance improvement program targeting severe sepsis. Crit Care Med 2010;38(02): 367-374

45 Ferrer R, Artigas A, Levy MM, et al; Edusepsis Study Group. Improvement in process of care and outcome after a multicenter severe sepsis educational program in Spain. JAMA 2008;299(19): 2294-2303

46 Seymour CW, Gesten F, Prescott HC, et al. Time to treatment and mortality during mandated emergency care for sepsis. $\mathrm{N}$ Engl J Med 2017;376(23):2235-2244

47 Cupit-Link MC, Nageswara Rao A, Warad DM, Rodriguez V. Lemierre syndrome: a retrospective study of the role of anticoagulation and thrombosis outcomes. Acta Haematol 2017 137(02):59-65

48 Vanassche T, Peetermans WE, Herregods MC, Herijgers $P$ Verhamme P. Anti-thrombotic therapy in infective endocarditis. Expert Rev Cardiovasc Ther 2011;9(09):1203-1219

49 Halton JML, Picard AC, Harper R, et al. Pharmacokinetics, pharmacodynamics, safety and tolerability of dabigatran etexilate oral liquid formulation in infants with venous thromboembolism. Thromb Haemost 2017;117(11):2168-2175

50 Young G, Yee DL, O'Brien SH, Khanna R, Barbour A, Nugent DJ. FondaKIDS: a prospective pharmacokinetic and safety study of fondaparinux in children between 1 and 18 years of age. Pediatr Blood Cancer 2011;57(06):1049-1054

51 Willmann S, Becker C, Burghaus R, et al. Development of a paediatric population-based model of the pharmacokinetics of rivaroxaban. Clin Pharmacokinet 2014;53(01):89-102

52 Malec L, Young G. Treatment of venous thromboembolism in pediatric patients. Front Pediatr 2017;5:26

53 Kearon C, Akl EA, Ornelas J, et al. Antithrombotic therapy for VTE disease: CHEST Guideline and Expert Panel Report. Chest 2016; 149(02):315-352

54 Redford MR, Ellis R, Rees CJ. Fusobacterium necrophorum infection associated with portal vein thrombosis. J Med Microbiol 2005;54(Pt 10):993-995

55 Schulman S, Kearon C; Subcommittee on Control of Anticoagulation of the Scientific and Standardization Committee of the International Society on Thrombosis and Haemostasis. Definition of major bleeding in clinical investigations of antihemostatic medicinal products in non-surgical patients. J Thromb Haemost 2005;3(04):692-694

56 Jackson D, Daly J, Saltman DC. Aggregating case reports: a way for the future of evidence-based health care? Clin Case Rep 2014 2(02):23-24

57 Vazquez FJ, Paulin P, Rodriguez P, Lubertino M, Gándara E. The outcome of pulmonary vein thrombosis in non-surgical patients. A systematic review and case report. Thromb Haemost 2015; 113(05):1151-1154

58 Khalil N, Nicotra A, Rakowicz W. Treatment for meralgia paraesthetica. Cochrane Database Syst Rev 2012;12:CD004159

59 Buonfrate D, Requena-Mendez A, Angheben A, et al. Severe strongyloidiasis: a systematic review of case reports. BMC Infect Dis 2013;13:78 
60 Cardona Zorrilla AF, Reveiz Herault L, Casasbuenas A, Aponte DM Ramos PL. Systematic review of case reports concerning adults suffering from neutropenic enterocolitis. Clin Transl Oncol 2006 8(01):31-38

61 George JN, Raskob GE, Shah SR, et al. Drug-induced thrombocytopenia: a systematic review of published case reports. Ann Intern Med 1998;129(11):886-890
62 Abdel-Wahab N, Lopez-Olivo MA, Pinto-Patarroyo GP, SuarezAlmazor ME. Systematic review of case reports of antiphospholipid syndrome following infection. Lupus 2016;25(14):1520-1531

63 Stewart LA, Clarke M, Rovers M, et al; PRISMA-IPD Development Group. Preferred Reporting Items for Systematic Review and Meta-Analyses of individual participant data: the PRISMA-IPD Statement. JAMA 2015;313(16):1657-1665 